\title{
Surgical mortality in an aneurysm population: effects of age, blood pressure and preoperative neurological state
}

\author{
L ARTIOLA i FORTUNY, C B T ADAMS, AND M BRIGGS \\ From the Department of Neurological Surgery, The Radcliffe Infirmary, Oxford
}

SUMMARY The mortality of 256 patients surgically treated for ruptured intracranial aneurysms was assessed with regard to age, systemic blood pressure on admission and preoperative neurological state. Increasing age, hypertension, and a poor neurological state (both singly and in combination) were found to influence surgical results dramatically. It is suggested that preoperative treatment of hypertension, and delay of operation in those patients aged over 50 years or hypertensive, as well as waiting for the neurological state to improve prior to operation, may lead to better results.

The immediate mortality of subarachnoid haemorrhage (SAH) secondary to ruptured intracranial aneurysms is $43 \%$. The studies by McKissock and his colleagues ${ }^{123}$ comparing conservative and surgical treatments at six months showed that the overall results were not improved by surgery (mortality rate $35 \%$ ). However, Pakarinen's ${ }^{4}$ study showed that those survivors from a first SAH who were managed conservatively, had a $35 \%$ mortality within a year and a $51 \%$ mortality within five years, the remainder dying subsequently at a rate of $3.3 \%$ per year. As the rate of rebleeding continues to be high in conservatively treated patients beyond six months after the first SAH, it is now generally accepted that surgery is justifiable, as long as the operative mortality is lower than the natural one.

Since the introduction of the operating microscope, the results of surgery for intracranial aneurysms reported by various authors have varied widely. The mortality has ranged between $3 \%,{ }^{5} 4 \cdot 3 \%,{ }^{6} 6 \%{ }^{7}$ and $15 \% .^{8}$ We present the operative results on a series of 256 patients with ruptured intracranial aneurysms. Selection was carried out exclusively on preoperative neurological status. The importance of various clinical factors in combination for prognosis has been

Address for reprint requests: Dr CBT Adams, Department of Neurological Surgery, The Radcliffe Infirmary, Oxford OX2 6HE, England.

Accepted 20 June 1980 discussed elsewhere. Here we are emphasising the results when age, blood pressure on admission, and neurological status are considered singly or in combination.

\section{Patient population}

The series consisted of 256 consecutive cases treated in Oxford during a five and a half year period. The patients ranged in age from 17 to 71 years with a mean age of $46 \cdot 6$ years. There were $113(44 \cdot 1 \%)$ men and $143(55.9 \%)$ women. All patients were admitted for subarachnoid haemorrhage (SAH). The interval between SAH and craniotomy ranged from one to 99 days with a mean of 13.7 days. All patients had single or multiple aneurysms demonstrated by arteriography. Aneurysm loci were grouped in the following manner: anterior communicating artery $(28.9 \%)$, middle cerebral artery $(31 \cdot 2 \%)$, internal carotid and posterior communicating arteries $(32 \cdot 0 \%)$, anterior cerebral artery $(2 \cdot 7 \%)$ and vertebro-basilar complex $(5 \cdot 1 \%)$. Three surgeons performed the operations and they differed in their personal choice of anaesthetic techniques. Most patients $(87.9 \%)$ had their aneurysms clipped. When simple clipping was not possible, aneurysms were treated by wrapping with muscle or cotton wool or both and cyanoacrylate glue in order to induce fibrosis and avoid further bleeding. Only patients in Botterell grades I, II and III were surgically treated.10 For the analysis of results, patients were subdivided according to their systemic blood pressure on admission. Some authors have reported that immediately after SAH, systemic hypertension is common. ${ }^{11}$ Since our patients were admitted to this department three to seven days after the initial 
bleed, it is probable that the acute hypertension present immediately after SAH had by that time subsided. We recognise, however, that our method of classification may have placed patients with chronic hypertension (but controlled on treatment) in the normotensive group. Also in this study we have not attempted to diagnose essential hypertension retrospectively on the basis of cardiac enlargement.

\section{Results}

Fifty-eight patients died between the time of operation and five years later. In 50 of these cases- $(19.5 \%)$ of the total population-death could be attributed directly to the aneurysm. The remaining deaths were due to unrelated causes (carcinoma, accident, cardiovascular disease). As these eight patients had all resumed premorbid activities before they died, and as there was no reason to believe that their aneurysms would have given them further trouble, they were included in the group of survivors for the analysis of mortality.

Table 1 summarises the operative results when age, systemic blood pressure and preoperative neurological grade are considered in isolation. The mortality rates increased with age, presence of hypertension and worsening preoperative grade. None of the patients under 30 died. When these three factors were considered in combinations of two it emerged that the presence of hypertension had a great influence on results regardless of age. Indeed, the mortality rate in hypertensive patients over and under 50 years of age was virtually identical: it was sixfold higher than in young (under 50 years) normotensive patients and almost one third higher than in older (over 50 years) normotensive patients (table 2).

When age was considered with preoperative neurological grade the results showed that young (under 50 years) patients in grade I had a mortality rate of $9.1 \%$ while $10 \%$ of young patients in grade II died. On the other hand, young patients in grade III showed a mortality four times higher than the combination of young patients in the better neurological grades.

Patients over 50 years in grade I did fairly well (mortality $12.3 \%$ ) whilst older patients in grades II and III showed very high mortality rates (ie nearly five times higher than that of their contemporaries in grade I) (table 3).

The combination of blood pressure and preoperative neurological condition, regardless of age, yielded the following dramatic results (table 4): the presence of hypertension in any neurological state at least doubled the mortality. In addition, in the presence of hypertension, patients in both grades II and III did very badly.

When age, blood pressure and preoperative neurological state were considered together (table 5), it was found that of the 75 patients in optimum condition (that is under 50 years, normotensive, grade I), only three $(4.0 \%)$ died. These three patients developed generalised vasospasm postoperatively. Conversely, normotensive, grade I patients over 50 years showed a $14.3 \%$ mortality rate. Young normotensive patients in

Table 2 Operative mortality, age, and blood pressure

\begin{tabular}{lll}
\hline \multicolumn{3}{c}{$\%$ Mortality } \\
\hline Blood pressure & Age $(y r)$ \\
\hline Under 50 & Over 50 \\
\hline Normal $<140 / 90 \mathrm{~mm} \mathrm{Hg}$ & $\frac{5}{98}=5.1 \%$ & $\frac{15}{64}=23.4 \%$ \\
Hypertension $>140 / 90 \mathrm{~mm} \mathrm{Hg}$ & $\frac{12}{37}=32.4 \%$ & $\frac{18}{57}=31.5 \%$ \\
\hline
\end{tabular}

Table 1 Operative results

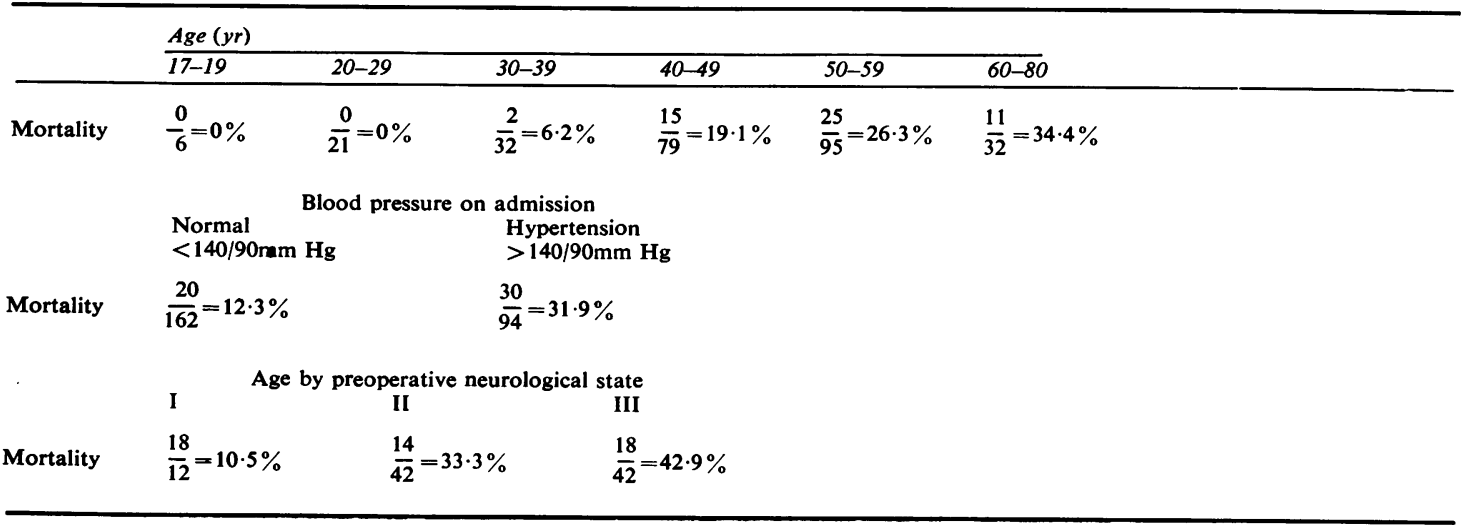


grade II and III had a combined mortality rate of $8.6 \%$ as opposed to a $40.9 \%$ rate in older, normotensive patients in the same Botterell grades. The highest mortality rate was found in

Table 3 Operative mortality, age, and neurological grade

\begin{tabular}{lcl}
\hline \multicolumn{3}{c}{$\%$ Mortality } \\
\hline Botterell neurological grade & \multicolumn{1}{c}{ Age } \\
\hline Under 50 & Over 50 \\
I & $\frac{9}{99}=9 \cdot 1 \%$ & $\frac{9}{64}=12 \cdot 3 \%$ \\
II & $\frac{2}{20}=10 \cdot 0 \%$ & $\frac{12}{22}=54 \cdot 5 \%$ \\
III & $\frac{6}{16}=37.5 \%$ & $\frac{12}{14}=46 \cdot 1 \%$ \\
\hline
\end{tabular}

Table 4 Operative mortality, blood pressure, and neurological grade

\begin{tabular}{lll}
\hline \multicolumn{3}{c}{$\%$ Mortality } \\
\hline Botterell neurological grade & $\frac{9}{\text { Blood pressure }}$ \\
\hline I & $\frac{9}{117}=7 \cdot 7 \% \quad \frac{9}{55}=16.4 \%$ \\
II & $\frac{4}{23}=17 \cdot 4 \% \quad \frac{10}{19}=52.6 \%$ \\
III & $\frac{7}{22}=31 \cdot 8 \% \quad \frac{11}{20}=55.0 \%$ \\
\hline
\end{tabular}

Table 5 Operative mortality, age, blood pressure, and neurological grade

\begin{tabular}{lccl}
\hline \multicolumn{4}{c}{$\%$ Mortality } \\
\hline Blood pressure & Botterell grade & \multicolumn{1}{l}{ Age } & \\
\hline Under 50 & Over 50 \\
\hline I & $\frac{3}{72}=4 \cdot 0 \%$ & $\frac{6}{36}=14 \cdot 3 \%$ \\
II & $\frac{1}{13}=7 \cdot 1 \%$ & $\frac{3}{6}=33 \cdot 3 \%$ \\
Hypertension & III & $\frac{1}{8}=11 \cdot 1 \%$ & $\frac{6}{7}=46 \cdot 1 \%$ \\
& I & $\frac{6}{18}=25 \cdot 0 \%$ & $\frac{3}{28}=9 \cdot 7 \%$ \\
& II & $\frac{1}{5}=16 \cdot 7 \%$ & $\frac{9}{4}=69 \cdot 2 \%$ \\
& III & $\frac{5}{2}=71 \cdot 4 \%$ & $\frac{6}{7}=46 \cdot 1 \%$ \\
\hline
\end{tabular}

older, hypertensive patients in grades II and III $(57.7 \%)$. Younger hypertensive patients in the same grades did somewhat better (mortality $46 \cdot 1 \%)$.

It will be noted that the death rate in hypertensive patients over 50 years in grade I was lower than that of both normotensive patients over 50 years and that of hypertensive patients under 50 in grade $\mathrm{I}$.

\section{Discussion}

The importance of age in prognosis is well recognised. ${ }^{812}$ Our results show that even in the best of circumstances (that is no hypertension and grade I) the mortality rate of patients over 50 years is three times as high as that of younger patients in similar clinical condition. The effect of hypertension and poor preoperative neurological state on surgical results have also been reported elsewhere. ${ }^{812}$ However, this study emphasises its importance in both young and older patients. A mortality rate of $32 \%$ in both hypertensive age groups warrants the treatment of hypertension prior to surgery in an attempt to see if results might improve. It is evident that normotensive patients under 50 years benefit from surgery even if their neurological state is not optimum (grades II and III). However, older normotensive patients in these grades do poorly. Surgery is justifiable in such patients only if the mortality rate is lower than the expected mortality for conservative treatment (that is $51 \%, 5$ years post $\mathrm{SAH}$ ). On the other hand, it is clear that both young and older hypertensive patients in less than optimal conditions do badly. Some authors have already reported on the beneficial effects of long-term hypotension for the definitive treatment of ruptured aneurysms. ${ }^{13} 14$ We therefore suggest delaying surgery for three or four weeks post SAH in patients over 50 years or in any patients with hypertension so as to reduce the likelihood of postoperative vasospasm occurring.

Inspection of the population data showed that the incidence of post-operative vasospasm in patients aged over 50 years in grade I with hypertension $(60.0 \%)$ was significantly lower than that of younger normotensives (75\%) and hypertensives $(80 \%)$ and that of older normotensives $(81.5 \%)$ in grade I. It is not clear why this should be the case. However, it is possible to conjecture that the atherosclerosis in older hypertensive patients might somehow prevent them developing vasospasm. This, of course, remains to be confirmed. The significance of spasm on mortality has been discussed elsewhere. 
It is important to note that in this series increasing age, worsening Botterell grade and hypertension did not predispose the patient to spasm, either singly or in combination. It appears, however, that in the presence of hypertension and poor preoperative state, the patient is less able to compensate for vasospasm.

These results explain the wide variations in operative mortality for this condition. On the basis of these figures we intend to delay surgery for three to four weeks in patients either over 50 years or hypertensive at any age. During this time we will prescribe an antifybrinolytic drug and treat any hypertension. In normotensive patients under 50 years of age we will continue to operate, if grade I or II, at about 10 days after the bleed. Whether this policy is justified will depend on the number of deaths occurring from rebleeding while waiting for operation and we intend to report our results based on the above policy in the future.

We wish to thank $\mathrm{Mr} R$ Gye for allowing us to include his patients in this study and Mrs Joan Smith for invaluable secretarial assistance. This study was supported by Medical Research Council Project Grant No. G977/184/N.

\section{References}

1 McKissock W, Richardson A, Walsh L. "Posterior Communicating" aneurysms. A controlled trial of the conservative and surgical treatment of ruptured aneurysms of the internal carotid artery at or near the joint of origin of the posterior communicating artery. Lancet 1960; 1:1203-6.

2 McKissock W, Richardson A, Walsh L. Middle cerebral aneurysms. Further results in the control trial of conservative and surgical treatment of ruptured intracranial aneurysms. Lancet 1962; 2:417-21.

3 McKissock W, Richardson A, Walsh L. Anterior communicating aneurysms. A trial of conservative and surgical treatment. Lancet 1965; 1:873-6.

4 Pakarinen S. Subarachnoid haemorrhage. Acta neurologica Scandinavica 1967; 43: Suppl 29.

5 Yasargil MG, Fox JL. The microsurgical approach to intracranial aneurysms. Surg Neurol 1975; 3:7-14.

6 Hollin SA, Decker RE. Microsurgical treatment of internal carotid artery aneurysms. J Neurosurg 1977; 47:142-29.

7 Krayenbühl HA, Yasargil MG, Flamm ES et al. Microsurgical treatment of intracranial saccular aneurysms. J Neurosurg 1972; 37:678-85.

8 Adams CBTA, Loach AB, O'Laoire SA. Intracranial aneurysms: analysis of results of microneurosurgery. Brit Med J 1976; 2:607-9.

9 Artiola i Fortuny L, Prieto-Valiente L. Long term prognosis in surgically treated intracranial aneurysms. I: Mortality. J Neurosurg 1980; In Press.

10 Botterell EH, Lougheed WM, Scott JW, Wandewater SL. Hypothermia, and interruption of carotid, or carotid and vertebral circulation in the surgical management of intracranial aneurysms. J Neurosurg 1956; 13:1-42.

11 Benedict CR, Loach AB. Clinical significance of plasma adrenaline and noradrenaline concentrations in patients with subarachnoid haemorrhage. J Neurol Neurosurg Psychiatry 1978; 41:113-7.

12 McKissock W, Paine K, Walsh L. An analysis of the results of treatment of ruptured intracranial aneurysms. J Neurosurg 1960; 17:762-7.

13 Slosberg PS. Treatment of ruptured intracranial aneurysms by induced hypotension. Mt Sinai j Med 1975; 40:82-90.

14 Post KD, Flamm ES, Goodgold A, Ranzohoff J. Ruptured intracranial aneurysms. Case morbidity and mortality. J Neurosurg 1977; 46:290-5. 


\section{Correction concerning:}

Surgical mortality in an aneurysm population: effects of age, blood pressure and preoperative neurological state

By L Artiola i Fortuny, C B T Adams, and $M$ Briggs. From the Department of Neurological
Surgery, The Radcliffe Infirmary, Oxford. (Journal of Neurology, Neurosurgery, and Psychiatry 1980; 43:879-82.)

The authors apologise for errors in tables 1, 3 and 5. These are printed below in correct form.

Table 1 Operative results

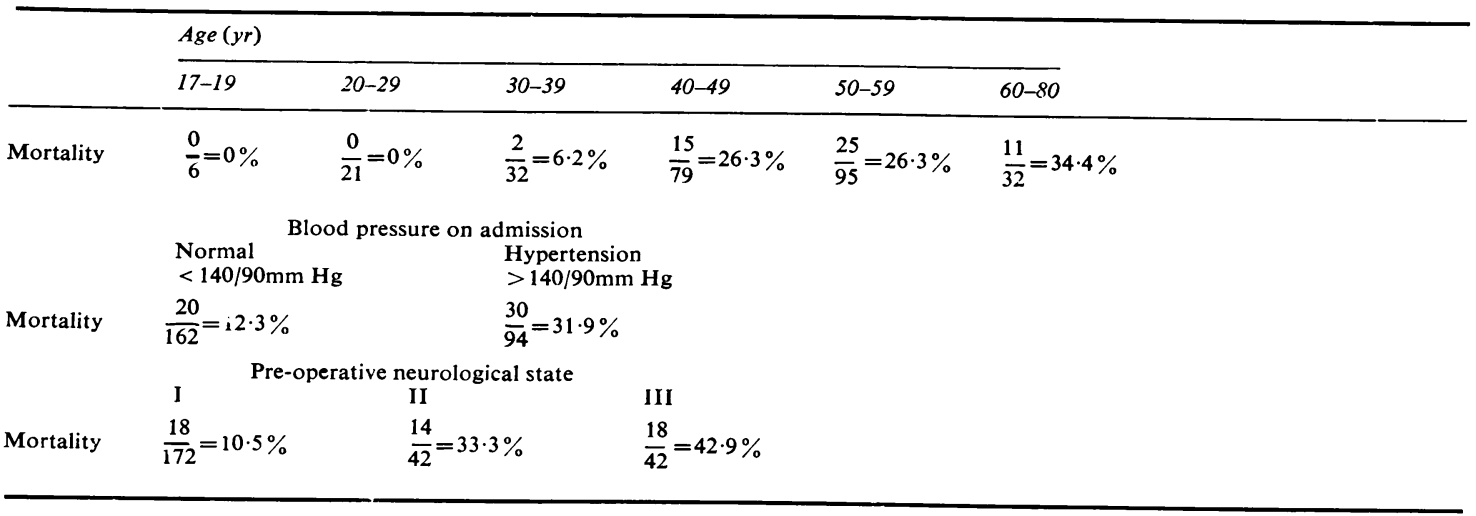

Table 3 Operative mortality, age, and neurological grade

\begin{tabular}{lll}
\hline \multicolumn{3}{c}{$\%$ Mortality } \\
\hline Botterell neurological grade & Ane \\
\hline Under $50 y r$ & Over $50 y r$ \\
II & $\frac{9}{99}=9 \cdot 1 \%$ & $\frac{9}{73}=12.3 \%$ \\
III & $\frac{2}{20}=10 \cdot 0 \%$ & $\frac{12}{22}=54.5 \%$ \\
\hline
\end{tabular}

Table 5 Operative mortality, age, blood pressure and neurological grade

\begin{tabular}{lccc}
\hline \multicolumn{4}{c}{$\%$ Mortality } \\
\hline Blood pressure & Botterell grade & Age \\
\hline Normal & I & $\frac{3}{75}=4.0 \%$ & $\frac{6}{42}=14 \cdot 3 \%$ \\
& II & $\frac{1}{14}=7 \cdot 1 \%$ & $\frac{3}{9}=33 \cdot 3 \%$ \\
Hypertension & III & $\frac{1}{9}=11 \cdot 1 \%$ & $\frac{6}{13}=46 \cdot 1 \%$ \\
& I & $\frac{6}{24}=25.0 \%$ & $\frac{3}{31}=9 \cdot 7 \%$ \\
& II & $\frac{1}{6}=16.7 \%$ & $\frac{9}{13}=69 \cdot 2 \%$ \\
& III & $\frac{5}{7}=71 \cdot 4 \%$ & $\frac{6}{13}=46 \cdot 1 \%$ \\
\hline
\end{tabular}

